\title{
Influência da polimerização por meio da energia de microondas sobre a porosidade interna de bases de resina acrílica de prótese total superior Influence of microwave polymerization method on the porosity of maxillary complete denture base resins
}

Ana Carolina PERO

Doutoranda - Programa de Pós-Graduação em Reabilitação Oral - Área de concentração - Prótese - Faculdade de Odontologia de Araraquara - UNESP - Araraquara - SP

\section{Ana Carolina BROGNA}

Cirurgiã - Dentista - Araraquara - SP

\section{Juliê MARRA}

Mestranda - Programa de Pós-Graduação em Reabilitação Oral - Área de concentração - Prótese - Faculdade de Odontologia de Araraquara - UNESP - Araraquara - SP

\section{Débora Barros BARBOSA}

Professor Doutor - Disciplina de Prótese Total - Departamento de Materiais Odontológicos e Prótese - Faculdade de Odontologia de Araçatuba - UNESP - Araçatuba - SP

\section{Marco Antonio COMPAGNONI}

Professor Titular - Disciplina de Prótese Total - Departamento de Materiais Odontológicos e Prótese - Faculdade de Odontologia de Araraquara - UNESP - Araraquara - SP

\begin{abstract}
Resumo
A porosidade na resina acrílica (RA) é atribuída a uma série de fatores e pode interferir nas propriedades deste material e na estética das bases protéticas. Ela pode estar associada à polimerização incompleta da RA ou ao excesso de aquecimento durante a sua polimerização. A utilização da energia de microondas na polimerização da RA é um processo que apresenta como principal vantagem a economia de tempo nos procedimentos laboratoriais para a confecção de próteses totais. No entanto, é importante que se utilizem tempos e potências adequados para que não ocorra prejuízo às propriedades físicas e mecânicas da RA. O objetivo do presente trabalho foi verificar a presença de poros em diferentes regiões de bases protéticas de RA de prótese total superior, com espessura de $3,5 \mathrm{~mm}$, processadas em três ciclos de polimerização por meio da energia de microondas: A) $500 \mathrm{~W}$ por 3'; B) $90 \mathrm{~W}$ por 13' (mufla na vertical) $+500 \mathrm{~W}$ por 90 " (mufla na horizontal); C) $320 \mathrm{~W}$ por 3' + 4' $0 \mathrm{~W}+720 \mathrm{~W}$ por 3'. O grupo controle foi polimerizado em banho de água quente por $9 \mathrm{~h}$ a $74 \mathrm{oC} \mathrm{(ciclo} \mathrm{T).} \mathrm{Após} \mathrm{o} \mathrm{seu} \mathrm{processamento,} \mathrm{as} \mathrm{bases} \mathrm{foram} \mathrm{seccionadas} \mathrm{transversalmente} \mathrm{em} \mathrm{três} \mathrm{regiões} \mathrm{(anterior,}$ média e posterior) e em cada região três áreas foram analisadas. As imagens dessas áreas foram capturadas em um microscópio e a área dos poros foi quantificada por um programa de computador (Leica). O teste de Kruskal-Wallis não detectou diferenças entre os diferentes ciclos e regiões das bases avaliadas $(\alpha=.05)$. Nenhuma diferença significativa na porosidade foi encontrada em relação às regiões analisadas nas bases polimerizadas nos ciclos em microondas. Ainda, a quantidade de poros nas bases polimerizadas em microondas foi semelhante para as bases polimerizadas pelo método convencional, independente da região analisada. Concluiu-se que a presença de poros não sofreu influência do ciclo de polimerização e da área da base protética maxilar analisada.
\end{abstract}

\section{UNITERMOS}

Porosidade; microondas, prótese total.

\section{INTRODUÇÃO}

A porosidade na resina acrílica é uma característica indesejável que pode ser causada por diversos fatores, como a proporção pó/líquido incorreta, falta de agregação das partículas do pó no líquido, inclusão da resina em estágio inadequado e utilização de ciclos de polimerização inadequados ${ }^{18}$. Alguns autores ${ }^{15,20}$ observaram que se o ar incorporado à resina durante sua manipulação não for completamente liberado, e isso, adicionado à contração de polimerização, são fatores decisivos na geração de poros.

Para Davenport ${ }^{9}$ a presença de poros na resina acrílica, além de alterar suas propriedades físicas, pode 
causar a estomatite protética, que se desenvolve devido a proliferação de Cândida albicans presente na placa bacteriana contida no interior dos poros.

O primeiro experimento utilizando a energia de microondas para polimerização de resinas acrílicas foi realizado por $\mathrm{Nishii}^{19} \mathrm{em} \mathrm{1968.} \mathrm{O} \mathrm{autor} \mathrm{buscava}$ reduzir a quantidade de porosidades internas nas bases protéticas de resina acrílica, relatando como principais vantagens desse método de processamento, grande economia de tempo e aumento do grau de polimerização.

Nos anos seguintes, a energia de microondas foi novamente reportada por Kimura ${ }^{16}$, em 1983, dando sequiência a uma série de outras pesquisas. $\mathrm{O}$ autor salientou outras vantagens em se utilizar a energia de microondas, como mínimas alterações de cor na resina, redução das trincas nos dentes artificiais e nas bases de resina, além de melhor adaptação da base protética. Além disso, a energia de microondas forneceu aquecimento por igual da resina acrílica durante a polimerização.

De Clerck ${ }^{10}$, em 1987, explorou amplamente a utilização da energia de microondas na polimerização de resinas acrílicas utilizadas em próteses orais. $\mathrm{O}$ autor explicou que as microondas geradas pelo magnetron a uma frequência de $2450 \mathrm{MHz}$ fazem com que as moléculas de metilmetacrilato girem em torno de si mesmas cerca de 5 bilhões de vezes por segundo. Conseqüentemente, ocorrem várias colisões intermoleculares que causam rápido aquecimento da resina. Já no método convencional em banho de água, o aquecimento da resina ocorre passivamente porque o calor é gerado externamente e as moléculas de monômero se chocam em conseqüência desse aquecimento.

A fim de favorecer as propriedades físicas e mecânicas desse material por meio de um ciclo de polimerização efetivo na confecção de próteses totais, diversos estudos têm sido realizados ${ }^{2,4,8,12,13,17,24}$. Em muitos desses estudos, as propriedades das resinas acrílicas foram comparadas quando polimerizadas em microondas e em ciclo convencional em banho de água quente, e demonstraram resultados semelhantes ${ }^{1,2,4}$, 6-8,10,17-19,22,25.

Ao avaliar a quantidade de poros de uma resina acrílica termopolimerizável sob influência de diferentes ciclos de polimerização em banho de água quente e por meio da energia de microondas por 3 minutos a $500 \mathrm{~W}$, Borges et al. ${ }^{7}$ não encontraram diferença entre o ciclo convencional e o de microondas, mesmo não utilizando resina acrílica específica para este último método de polimerização.
Yannikakis et al. ${ }^{30}$ avaliaram a quantidade de poros em dois tipos de resinas acrílicas (termopolimerizável convencional e específica para microondas) por meio de um método fotográfico. Espessuras de $3 \mathrm{~mm}$ e 6 $\mathrm{mm}$ de resinas acrílicas foram polimerizadas em dois ciclos no forno de microondas, sendo um curto, e outro longo. A maior quantidade de poros foi observada na resina acrílica termopolimerizável com espessura de $6 \mathrm{~mm}$, polimerizada em ciclo curto por meio da energia de microondas. Apesar disso, os ciclos de polimerização não apresentaram efeito significativo sobre a quantidade de poros de ambos os tipos de resina avaliados. Diante dos resultados obtidos, os autores alertaram que, apesar da polimerização por meio da energia de microondas ser uma opção atrativa quando o tempo é um fator crítico, deve ser utilizada com cautela para que não ocorram prejuízos às propriedades da resina acrílica.

No estudo realizado por Compagnoni et al. ${ }^{8}$ a quantidade de poros foi avaliada em espécimes de resina acrílica polimerizados em diferentes ciclos por microondas. A porosidade foi calculada a partir de fórmulas aritméticas que associavam o volume dos espécimes antes e após imersão em água ${ }^{4}$.Os autores não encontraram diferença ao analisar a quantidade de poros entre espécimes de resina acrílica polimerizados com 3 diferentes ciclos em microondas, e também não houve diferença entre os espécimes polimerizados em microondas e pelo método convencional em banho de água.

No entanto, Ilbay et al..$^{13}$ observaram que a porosidade foi superior na resina acrílica polimerizada em microondas sob altas potências. Reitz et al. ${ }^{22}$ também observaram que alta potência (400W) com ciclo curto (5 minutos) produziu maior quantidade de poros em espécimes mais espessos, que em baixa potência (90W) associada a maior tempo (13 minutos).

Segundo Harman \& Pittsburg ${ }^{11}$, para conseguir-se um ciclo de polimerização efetivo, deve-se considerar além da massa e forma do corpo-de-prova, a temperatura ambiente e a velocidade do aumento da temperatura durante a polimerização da resina acrílica. Jerolimov et al. ${ }^{14}$ afirmaram que, na avaliação da quantidade de poros, a variação na espessura e geometria de uma prótese são fatores que afetam a eficiência da dissipação do calor proveniente da reação exotérmica de polimerização, o que influenciaria na geração de poros.

Dessa forma, deve-se considerar a influência do ciclo de polimerização sobre a formação de poros na resina acrílica, principalmente quando se depara com diferentes métodos de polimerização e tipos de resina acrílica. 
Assim, o objetivo deste trabalho foi verificar a presença de poros em diferentes regiões em bases protéticas de resina acrílica de prótese total superior, confeccionadas em diferentes ciclos de polimerização por meio da energia de microondas em relação ao ciclo de polimerização convencional em banho de água quente.

Dessa forma, a hipótese nula do trabalho é de que os diferentes ciclos de polimerização estudados pro- duzem efeitos semelhantes sobre a presença de poros na resina acrílica avaliada, independente da região da base protética.

\section{Material e método}

A presença de poros foi avaliada em diferentes regiões da base de prótese total superior, em função de diferentes ciclos de polimerização (Tabela 1).

Tabela 1 - Método e ciclo de polimerização com a respectiva resina acrílica utilizada

\begin{tabular}{|c|c|c|}
\hline & Método e Ciclo de Polimerização & Resina Acrílica \\
\hline CICLO A & Microondas: 3 minutos a 500W 16 & Onda-Cryl (específica para microondas) \\
\hline CICLO B & $\begin{array}{l}\text { Microondas: } 13 \text { minutos a 90W (mufla na vertical) + } \\
90 \text { segundos a } 500 \mathrm{~W} \text { (mufla na horizontal) } 26\end{array}$ & Onda-Cryl (específica para microondas) \\
\hline CICLO C & $\begin{array}{l}\text { Microondas: } 3 \text { minutos a } 320 \mathrm{~W}+4 \text { minutos } 0 \mathrm{~W}+ \\
3 \text { minutos a } 720 \mathrm{~W}^{*}\end{array}$ & Onda-Cryl (específica para microondas) \\
\hline CICLO T & Banho de água quente: 9 horas a $74^{\circ} \mathrm{C}^{28}$ & Clássico (termopolimerizável convencional) \\
\hline
\end{tabular}

* Ciclo de polimerização recomendado pelo fabricante da resina acrílica Onda-Cryl.

\section{Obtenção dos espécimes}

O espécime correspondeu a uma base protética em resina acrílica confeccionada sobre um modelo funcional de gesso pedra tipo IV (Herostone, Vigodent S/A Ind e Com., Rio de Janeiro, RJ) obtido a partir de um rebordo desdentado total maxilar padrão. A base apresentava 3,5 $\mathrm{mm}$ de espessura e foi obtida primeiramente em cera 7 com auxílio de uma matriz de silicone (Figura 1). Essa matriz permitiu a padronização da forma e espessura das bases confeccionadas em cera. A espessura da base protética em cera também foi controlada com uso de sonda milimetrada posicionada em diferentes regiões desta base.

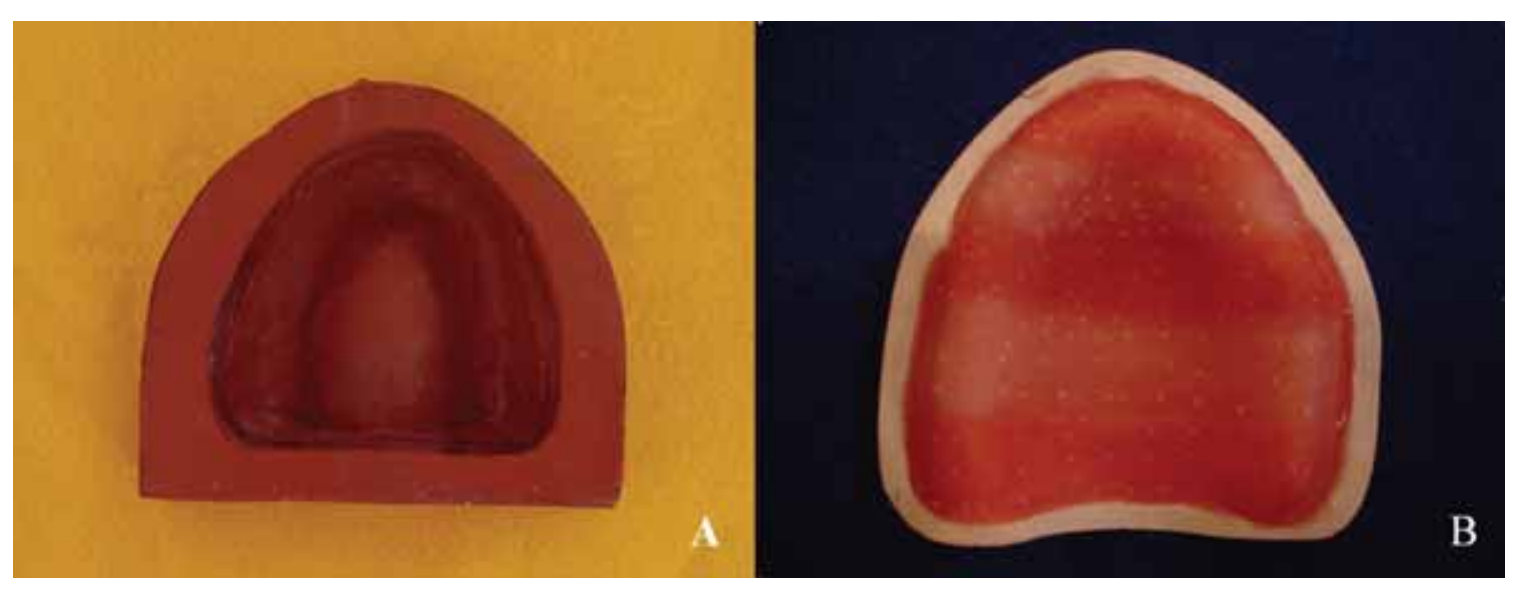

FIGURA 1 - Matriz de silicona para o enceramento do modelo (A) e espécime encerado (B). 
Cada espécime foi incluído em mufla com gesso pedra tipo III (Herodent, Vigodent S/A Ind e Com., Rio de Janeiro, RJ) e, após a eliminação da cera, a resina acrílica foi prensada gradualmente em prensa hidráulica de bancada e mantida sob pressão durante 30 minutos $^{3,5}$. A seguir, procedeu-se com a polimerização (Tabela 1, pág.5), resfriamento da mufla sob bancada e desinclusão do espécime. Foi dado acabamento com fresa Maxi-cuti (Edenta AG - Dentalprodukte, Au/SG, Switzerland) e lixas para acabamento (Norton A237, Comercial e Técnica de Abrasivos Ltda, Campinas, SP) e a espessura da base em resina acrílica foi certificada em diferentes regiões com espessímetro (Golgran Ind. e Com. de Instrumental Odontológico Ltda, Pirituba, SP).

Foram confeccionados 10 espécimes para cada ciclo de polimerização, totalizando uma amostra de 40. Cada espécime (Figura 2A) foi, então, seccionado transversalmente com disco de carborundum em três regiões (Figura $2 \mathrm{C}$ ) já demarcadas no modelo funcional padrão, como mostra a Figura 2 B. As faces seccionadas receberam acabamento com lixas de granulações decrescentes $(400,600$ e 1000) para permitir a visualização dos poros nestas faces. Essas regiões foram identificadas como: P - região posterior da base da prótese, seguindo-se uma linha que passa sobre a parte média do túber direito indo em direção ao túber esquerdo; $\mathrm{M}$ - região média da base da prótese, onde um corte será realizado seguindo-se uma linha que passa na região de molares, atravessa o palato e estende-se até a região de molares do outro lado e A - região mais anterior, correspondente a uma área retilínea transversal à base, que se estende de canino a canino, passando pelo palato.

Para cada região da base da prótese $(\mathrm{P}, \mathrm{M} \mathrm{e} \mathrm{A})$, três áreas (1, 2 e 3$)$ foram selecionadas para a análise da porosidade (Figura $2 \mathrm{D}$ ), totalizando nove setores de observação para cada uma das bases de prótese.

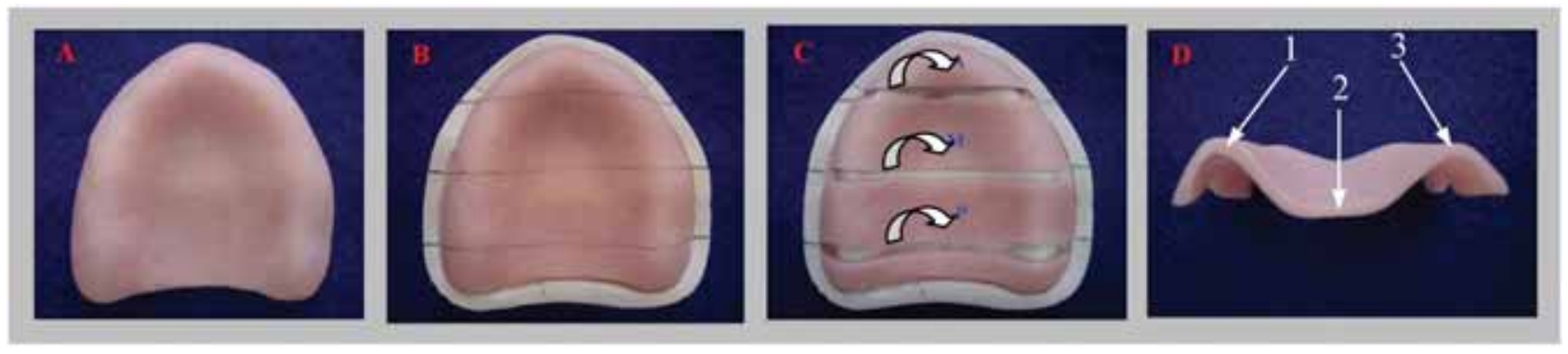

FIGURA 2. A) Base da prótese total superior em resina acrílica; B) Base sobre o modelo com traçado à lápis, indicando onde será seccionada a base; C) Base já seccionada nas linhas demarcadas evidenciando-se as regiões das faces seccionadas onde foram realizadas as análises da porosidade (regiões $\mathrm{P}$, M e A) e D) Num corte transversal, uma das partes da base seccionada com as setas indicando as regiões que foram analisadas.

A presença de poros foi avaliada por meio de um programa de computador (Image Processing Analysis System - Leica Imaging Systems Ltd, Cambridge, London) que selecionou por meio de contraste de cores as áreas correspondentes aos poros internos na base da prótese total superior e as quantificou em $\mathrm{mm}^{2}$. O computador que contém o programa Leica é acoplado a um microscópio óptico, através do qual as regiões no espécime $(1,2$ e 3$)$ foram selecionadas para análise. Foi realizada, então, a captura das imagens pelo programa com lentes objetiva de $40 x$ e ocular de $0,63 x$.

A mensuração das áreas de poros foi feita com o auxílio de uma rotina de automação dos dados, desenvolvida propriamente para o objetivo proposto, e devidamente compatível ao sistema. Um pixel na imagem correspondeu a $0,00539 \mathrm{~mm}$ e, por contraste de cores, foi possível quantificar as áreas de poros nas bases em $\mathrm{mm}^{2}$. 
INFLUÊNCIA DA POLIMERIZAÇÃO POR MEIO DA ENERGIA DE MICROONDAS SOBRE A POROSIDADE INTERNA DE BASES DE RESINA ACRÍLICA DE PRÓTESE TOTAL SUPERIOR

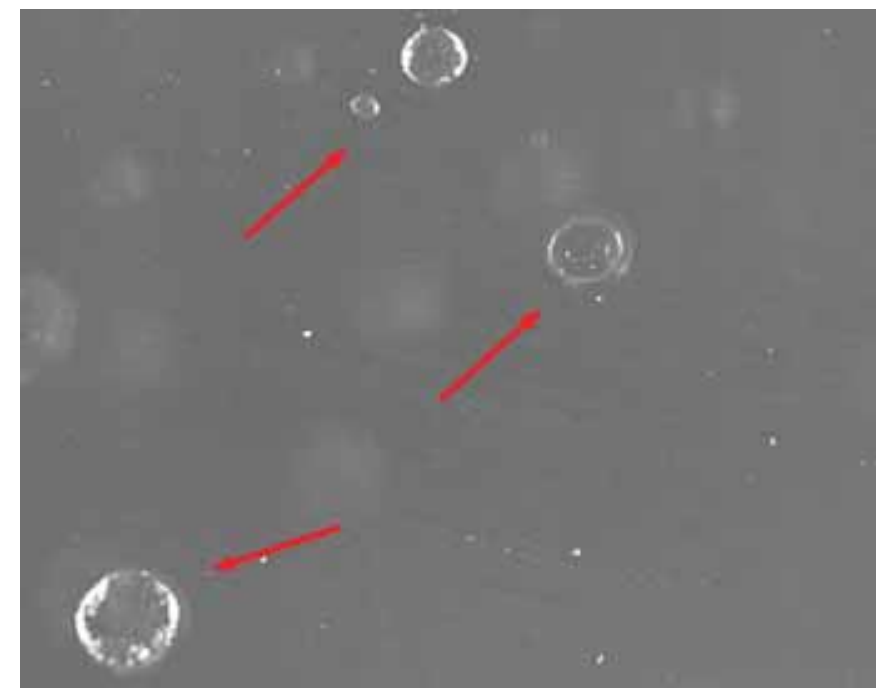

FIGURA 3. Imagem de uma região da resina acrílica capturada pelo microscópio, num aumento de $40 \mathrm{x}$. Os poros estão indicados por setas.

A diferença entre as diferentes séries de avaliação segundo a área, a região e o ciclo de polimerização, foi avaliada por meio do teste de Kruskal-Wallis. Comprovada a diferença, comparações post hoc não-paramétricas foram realizadas, avaliando-se os diferentes ciclos, de acordo com cada área da base protética (nove, no total). Todos os testes foram realizados com nível de significância de 0,05.

\section{Resultado}

Para os ciclos A e B, não foi observada porosidade em nenhum dos espécimes independente da região avaliada. Para o ciclo C, os resultados foram seme- lhantes, exceto em quatro espécimes: um apresentou porosidade de $0,23 \%$ na área 3 da região A, dois apresentaram poros na área 1 da região $\mathrm{P}(0,74 \%$ e 1,69\%) e um na área 2 da região $\mathrm{P}(0,02 \%)$. No ciclo $\mathrm{T}$, foi encontrada porosidade em apenas dois espécimes, um deles na área 2 da região $\mathrm{A}(0,06 \%)$ e o outro na área 2 da região $\mathrm{M}(0,04 \%)$.

Os resultados das diferentes combinações entre ciclos, regiões e áreas, totalizando 36 possibilidades, foram analisados por meio do teste de Kruskal-Wallis. Os postos médios observados para cada combinação estão representados no Gráfico 1, e o valor de de $\mathrm{P}$ obtido foi inferior a 0,001 .

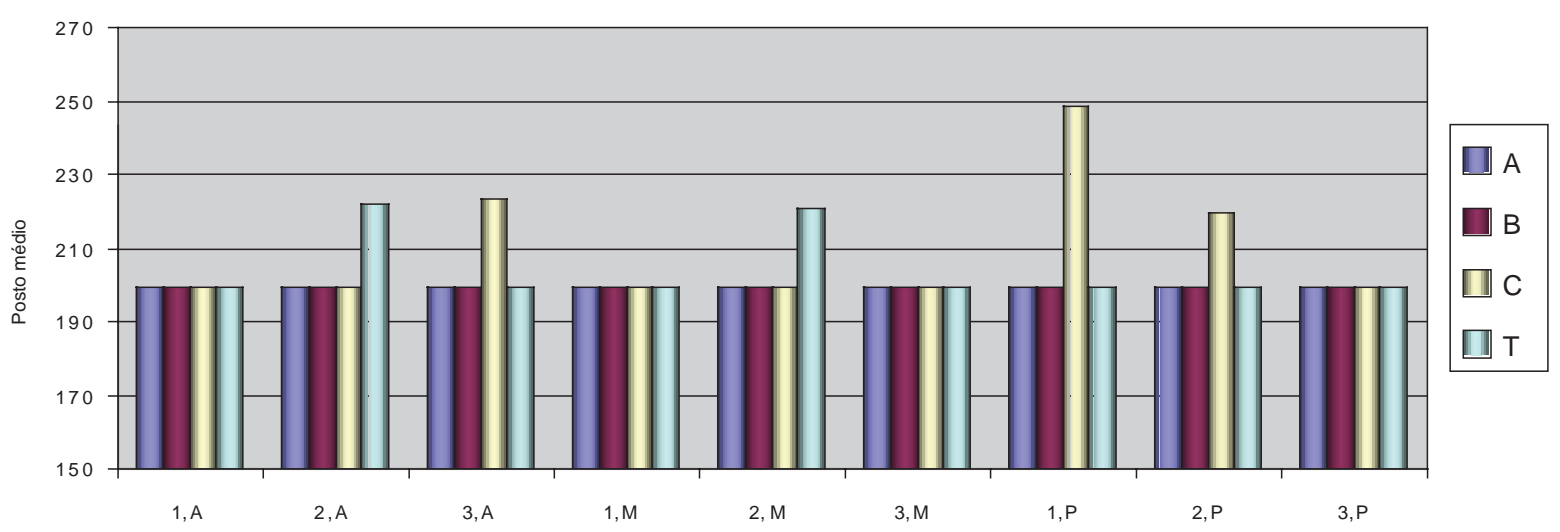

FIGURA 1 - Postos médios da porosidade para as diferentes combinações entre os quatro ciclos e os nove setores avaliados. 
A comparação post hoc não encontrou diferenças entre os ciclos para cada um dos setores exceto para a região 2, na área $\mathrm{A}$ (Tabela 2).

Tabela 2 - Resultados da comparação entre pares para a região 2 , área $A$.

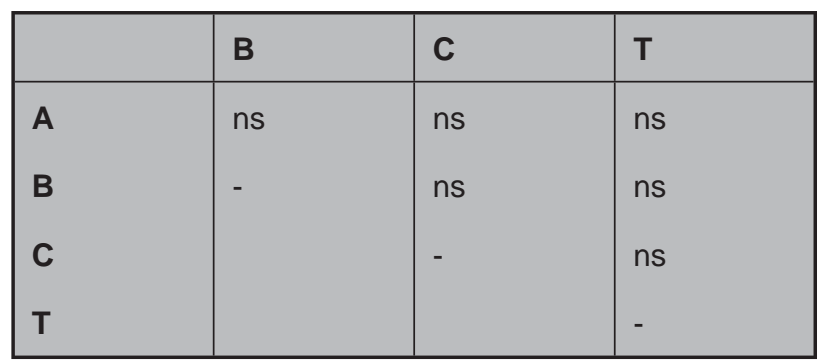

Diferença significante $\alpha=0,05$.

\section{Discussão}

A porosidade em bases protéticas de resina acrílica é um fenômeno complexo com origem multifatorial ${ }^{29}$. Ela pode estar relacionada com o tipo de material $^{2}$, ciclo de polimerização $0^{30}$, proporção pó/líquido incorreta da resina ${ }^{18} \mathrm{e}$ sua inclusão em estágio inadequado no interior da mufla ${ }^{23}$.

No presente estudo, a quantidade de poros encontrada na resina acrílica foi semelhante para os diferentes ciclos avaliados. Esses resultados estão de acordo com os trabalhos de Barbosa ${ }^{6}$, Bafile et al. ${ }^{4}$, Compagnoni et al..$^{8}$ e Ilbay et al. ${ }^{13}$. Ao utilizarem um método fotográfico de avaliação, Yannikakis et al. ${ }^{30}$ também observaram poucos poros quando a resina acrílica específica para microondas foi polimerizada independente do ciclo.

Truong \& Thomasz $^{27}$ observaram que a porosidade pode ser atribuída a um calor excessivo da reação exotérmica de polimerização e que pode ser reduzida através de um controle apropriado da energia fornecida no início da polimerização. No entanto, na presente pesquisa, a utilização do ciclo $\mathrm{B}$ em microondas, onde a polimerização iniciou-se com $90 \mathrm{~W}$ por 13 minutos e com uma pós-polimerização de $500 \mathrm{~W}$ por 90 segundos, forneceu resultados semelhantes em relação aos outros ciclos. Portanto, o controle da energia de microondas no início da polimerização, correspondente à primeira etapa do ciclo $\mathrm{B}$, não foi um fator influente na redução de poros nesse estudo, como observado por Truong \& Thomasz ${ }^{27}$.

Ao se comparar a porosidade dos espécimes de resina polimerizados por meio da energia de microondas e pelo método convencional em banho de água, verifi- cou-se também comportamento semelhante quanto à presença ou ausência de poros nos espécimes. Esses resultados estão de acordo com Bafile et al. ${ }^{4}$, Barbosa ${ }^{6}$, Compagnoni et al. ${ }^{8} \mathrm{e}$ Shlosberg et al. ${ }^{25}$ ao compararem também resinas acrílicas para base protética polimerizadas em microondas por 13 minutos a $90 \mathrm{~W}$ e mais 90 segundos a $500 \mathrm{~W}$ e em banho de água por 8 horas a $74^{\circ} \mathrm{C}$. Além disso, encontraram pequena quantidade de poros nas resinas polimerizadas em microondas, fato observado no presente estudo.

Apesar da análise estatística não ter encontrado diferença entre os grupos e as diferentes áreas da base avaliadas, algumas regiões desta base apresentaram poros em alguns ciclos de polimerização.

Para o ciclo convencional em banho de água (T), dois espécimes apresentaram poros localizados na região central da base de resina. Esses achados estão de acordo com outros trabalhos ${ }^{16,22,29}$ que também observaram maior concentração de poros no centro das amostras de resina acrílica. No entanto, no presente trabalho, foram observados poros nas extremidades de três bases polimerizadas no ciclo $\mathrm{C}$, e apenas uma apresentou poros no centro. Embora esses resultados não apresentem significância estatística, a localização dos poros poderia estar relacionada com o ciclo de polimerização, o tipo de resina utilizada e, especialmente, com a geometria do espécime ${ }^{14}$.

No presente estudo, a quantidade de poros foi avaliada por meio de análise visual através de microscopia ótica com aumento de 40x. Embora existam outros métodos para avaliação da presença de poros em resina acrílica ${ }^{4,6,8,21}$, onde a porosidade é calculada baseada na massa e no volume do espécime antes e após a sua imersão em água, o método utilizado nesse estudo é objetivo e fornece informações específicas e quantitativas sobre os poros nas regiões analisadas.

A presente pesquisa avaliou apenas uma marca de resina acrílica para base protética quanto à presença de poros. Além disso, apesar da conformação da base da prótese, sua espessura não variou. Portanto, são recomendáveis novos estudos com outras resinas acrílicas e ciclos de polimerização, assim como variações na espessura do espécime. Isso auxiliará na confecção de bases de próteses com menor quantidade de poros, melhor qualidade estética e maior longevidade dessas próteses.

Ainda, a hipótese nula do trabalho deve ser aceita pois os diferentes ciclos de polimerização produziram efeitos semelhantes sobre a porosidade na resina acrílica avaliada. 


\section{Conclusão}

Dentro das limitações desse estudo, concluiu-se que os diferentes ciclos de polimerização estudados produziram efeitos semelhantes sobre a presença de poros na resina acrílica avaliada, independente da região da base protética.

\section{Agradecimentos}

Ao Conselho Nacional de Desenvolvimento Científico e Tecnológico, ao Prof. Dr. João Aristeu da Rosa, da Faculdade de Ciências Farmacêuticas - UNESP, e a João Monti Junior, auxiliar de laboratório da Disciplina de Prótese Total da Faculdade de Odontologia de Araraquara-UNESP.

\begin{abstract}
Porosity in acrylic resin (AR) is attributed to a variety of factors and can interfere on properties of this material and aesthetics of denture bases. Porosity may be attributed can be associated to the incomplete polymerization of AR or excess of heating during polymerization. The main advantage of polymerizing denture base resin by microwave energy is a greatly reduced polymerization time. However, it is important to control the temperature accurately and ensure correct timing when microwave is used to minimize porosity in AR. The aim of this study was to investigate the occurrence of porosity in different areas of maxillary complete denture bases of AR processed by different polymerization cycles: A) $500 \mathrm{~W}$ for 3 minutes; B) $90 \mathrm{~W}$ for 13 minutes (vertical flask) + $500 \mathrm{~W}$ for 90 seconds (horizontal flask); C) $320 \mathrm{~W}$ for 3 minutes $+O W$ for 4 minutes $+720 \mathrm{~W}$ for 3 minutes. The control group was polymerized in water bath at $74^{\circ} \mathrm{C}$ for 9 hours (cycle T). After being processed, maxillary denture bases were cut so that 3 cross-sectional areas were formed $(A, M$, and $P)$. These surfaces were polished and selected under a microscope at $x 40$ magnification. The area of each pore was measured with a computer program (Leica), and the total area of pores per surface was calculated in mm 2 form. The Kruskal-Wallis test was used to compare porosity data $(\alpha=.05)$. There were no differences among the different cycles and surfaces of the maxillary bases evaluated. Microwave polymerization cycle had no effect on porosity $(P>.05)$. The amount of porosity in the microwave-polymerized bases was similar to the water bath-polymerized bases, regardless of the surface analyzed. With the limitations of this study, the occurrence of porosities was not influenced by the polymerization cycle and the area of maxillary denture base.
\end{abstract}

\title{
UNITERMOS
}

Porosity; microwaves; complete denture.

\section{REFERÊNCIAS}

1 Al Doori D, Huggett R, Bates JF. A comparison of denture base resins polymerized by microwave irradiation and by conventional water bath curing systems. Dent Mater. 1988 Feb.; 4(1):25-32.

2. Alkhatib MB, Goodacre CJ, Swartz ML, Munoz-Viveros CA, Andres CJ. Comparison of microwave-polymerized denture base resins. Int J Prosthodont 1990 May/June; 3(3):249-55.

3. Anusavice KJ. Phillips' science of dental materials. Pennsylvania: WB Saunders, 1996.

4. Bafile M, Graser GN, Myers ML, Li, EKH. Porosity of denture resin cured by microwave energy. J Prosthet Dent. 1991 Aug.;66(2):269-74.

5. Barbosa DB. Alteração da dimensão vertical de oclusão em próteses totais. Estudo comparativo entre diferentes ciclos de polimerização. Araraquara; 2000.

6. Barbosa DB. Avaliação da resistência à flexão e da porosidade de resina acrílica polimerizada em diferentes ciclos por meio da energia de microondas. Araraquara; 2003.

7. Borges LH, Domitti SS, Consani S. Influência de ciclos de polimerização sobre polimento, rugosidade, porosidade e dureza superficial da resina acrílica QC-20. Rev CROMG. 2000 maio/ago; 6(2): 68-77.

8. Compagnoni MA, Barbosa DB, Souza RF, Pero AC. The effect of polymerization cycles on porosity of microwave-processed denture base resin. J Prosthet Dent. 2004 Mar; 91(3):281-5.
9. Davenport JC. The oral distribution of candida in denture stomatitis. Br Dent J. 1970 Aug.; 129 (4):151-6.

10. De Clerck JP. Microwave polymerization of acrylic resins used in dental prostheses. J Prosthet Dent. 1987 May; 57(5):650-8.

11. Harman IM, Pittsburgh AB. Effects of time and temperature on polymerization of a methacrylate resin denture base. J Am Dent Assoc. 1949 Feb.; 38(2): 188-203.

12. Harrison A, Huggett R. Effect of the curing cycle on the residual monomer levels of acrylic resin denture base polymers. J Dent. 1992 Dec.; 20(2):370-4.

13. Ilbay SG, Güvener S, Alkumru HN. Processing dentures using a microwave technique. J Oral Rehabil. 1994 Jan; 21(1):103-9.

14. Jerolimov V, Brooks SC, Huggett R, Bates JF. Rapid curing of acrylic denture-base materials. Dent Mater 1989 Jan.; 5(1):18-22.

15. Keller JC, Lautenschlager EP. Porosity reduction and its associated effect on the diametral tensile strength of activated resins. J Prosthet Dent..1985 Mar.; 53(3):374-9.

16. Kimura H, Teraoka F, Ohnishi H, Saito T, Yato M. Applications of microwave for dental technique (Part 1). Doug-forming and curing of acrylic resins. J Osaka Univ Dent Sch. 1983 Dec.; 23:43-9.

17. Lai CP, Tsai MH, Chen M, Tay HH. Morphology and properties of denture acrylic resins cured by microwave energy and conventional water bath. Dent Mater. 2004 Feb.; 20(2):133-41.

18. Levin B, Sanders JL, Reitz PV. The use of microwave energy for processing acrylic resins. J Prosthet Dent. 1989 Mar.; 61(3):381-3. 
19. Nishii M. Studies on the curing of denture base resins with microwave irradiation: with particular reference to heat-curing resins. J Osaka Dent Univ. 1968; 2(1):23-40.

20. Ohashi M. Observations on the generating mechanism of internal porosity in polymerization of methyl methacrylic resin for dental use. J Nihon Univ Sch Dent 1961 Sept.; 1-13.

21. Pero, A.C. Avaliação da influência do método de polimerização e da espessura da base de próteses totais na porosidade da resina acrílica. Araraquara; 2005.

22. Reitz PV, Sanders JL, Levin B. The curing of denture acrylic resins by microwave energy. Physical properties. Quintessence Int. 1985 Aug.;16(8):547-51.

23. Samuel SMW, Gonzatto D, Suzuki RM. Avaliação da porosidade de resinas acrílicas de termo-polimerização. Rev Fac Odontol Porto Alegre. 1996 jul.; 37(1):18-20.

24. Sadamori S, Ganefiyanti T, Hamada T, Arima T. Influence of thickness and location on the residual monomer content of denture base cured by three processing methods. J Prosthet Dent 1994 July; 72(1):19-22.

25. Shlosberg SR, Goodacre CJ, Munoz CA, Schnell RJ. Microwave energy polymerization of poly(methyl methacrylate) denture base resin. Int J Prosthodont. 1989 Sept./Oct.; 2(5):453-8.

26. Takamata T, Setcos JC, Phillips RW, Boone ME. Adaptation of acrylic resin dentures as influenced by the activation mode of polymerization. J Am Dent Assoc. 1989; 119(2): 271-6.
27. Truong VT, Thomasz FGV. Comparison of denture acrylic resins cured by boiling water and microwave energy. Aust Dent J. 1988 May/June; 33(3):201-4.

28. Woeffel JB. Processing complete dentures. Dent Clin North Am 1977 Apr.; 21(2):329-38.

29. Wolfaardt JF, Cleaton-Jones P, Fatti P. The occurrence of porosity in a heat-cured poly (methyl methacrylate) denture base resin. J Prosthet Dent. 1986 Mar.; 55(3):393-400.

30. Yannikakis S, Zissis A, Polyzois G, Andreopoulos A. Evaluation of porosity in microwave-processed acrylic resin using a photographic method. J Prosthet Dent. 2002 June;87(6):613-9.

Recebido em: 25/07/06 Aprovado em: 05/01/07

Marco Antonio Compagnoni Faculdade de Odontologia de Araraquara UNESP - Departamento de Materiais Odontológicos e Prótese - Disciplina de Prótese Total Fone/ Fax: (16) 3301-6406 ou (16) 3301-6411

compagno@foar.unesp.br Rua Humaitá, 1680 - Centro Araraquara - SP 14801-903 